\title{
On a Periodic Predator-Prey System with Holling III Functional Response and Stage Structure for Prey
}

\section{Xiangzeng Kong, Zhiqin Chen, Li Xu, and Wensheng Yang}

Key Lab of Network Security and Cryptology, Fujian Normal University, Fuzhou, Fujian 350007, China

Correspondence should be addressed to Xiangzeng Kong, xzkong@fjnu.edu.cn

Received 14 October 2009; Accepted 4 March 2010

Academic Editor: Stephen Clark

Copyright (C) 2010 Xiangzeng Kong et al. This is an open access article distributed under the Creative Commons Attribution License, which permits unrestricted use, distribution, and reproduction in any medium, provided the original work is properly cited.

We propose and study the permanence of the following periodic Holling III predator-prey system with stage structure for prey and both two predators which consume immature prey. Sufficient and necessary conditions which guarantee the predator and the prey species to be permanent are obtained.

\section{Introduction}

The aim of this paper is to investigate the permanence of the following periodic stagestructure predator-prey system with Holling III functional response:

$$
\begin{aligned}
\dot{x}_{1}(t)= & a(t) x_{2}(t)-b(t) x_{1}(t)-d(t) x_{1}^{2}(t)-\frac{p_{1}(t) x_{1}^{2}(t)}{k_{1}(t)+x_{1}^{2}(t)} y_{1}(t) \\
& -\frac{p_{2}(t) x_{1}(t)}{k_{2}(t)+m(t) x_{1}(t)+n(t) y_{2}(t)} y_{2}(t), \\
\dot{x}_{2}(t)= & c(t) x_{1}(t)-f(t) x_{2}^{2}(t)-\frac{p_{3}(t) x_{2}^{2}(t)}{k_{3}(t)+x_{2}^{2}(t)} y_{2}(t), \\
\dot{y}_{1}(t)= & y_{1}(t)\left(-g_{1}(t)+\frac{h_{1}(t) x_{1}^{2}(t)}{k_{1}(t)+x_{1}^{2}(t)}-q_{1}(t) y_{1}(t)\right), \\
\dot{y}_{2}(t)= & y_{2}(t)\left(-g_{2}(t)+\frac{h_{2}(t) x_{1}(t)}{k_{2}(t)+m(t) x_{1}(t)+n(t) y_{2}(t)}+\frac{h_{3}(t) x_{2}^{2}(t)}{k_{3}(t)+x_{2}^{2}(t)}-q_{2}(t) y_{2}(t)\right),
\end{aligned}
$$


where $a(t), b(t), c(t), d(t), f(t), p_{i}(t), g_{i}(t), q_{i}(t), i=1,2$ and $h_{i}(t), k_{i}(t), i=1,2,3$, are all continuous positive $\omega$-periodic functions. Here $x_{1}(t)$ and $x_{2}(t)$ denote the density of immature and mature prey species, respectively, and $y_{i}$ is the density of the predators.

The periodic functions in (1.1) have the following biological meanings. The birth rate into the immature population is given by $a(t) x_{2}$; that is, it is assumed to be proportional to the existing mature population, with a proportionality coefficient $a(t)$. The death rate of the immature population is proportional to the existing immature population and to its square with coefficients $b(t)$ and $d(t)$, respectively. The death rate of the mature population is of a logistic nature, with proportionality coefficient $f(t)$. The transition rate from the immature individuals to the mature individuals is assumed to be proportional to the existing immature population, with proportionality coefficient $c(t)$. Similarly, $-g_{i}(t) y_{i}-q_{i}(t) y_{i}$ gives the densitydependent death rate of the predators. $p_{i}(t)$ and $h_{i}(t)$ give the coefficients that relate to the conversion rate of the immature prey biomass into predator biomass. More details about the biological background for (1.1) can be found in [1-10].

The function $p_{i}(t) x_{1}^{2}(t) /\left(k_{i}(t)+x_{1}^{2}(t)\right)$ represents the functional response of predator to immature prey. Let $\varphi_{i}\left(t, x_{1}\right)=p_{i}(t) x_{1}^{2}(t) /\left(k_{i}(t)+x_{1}^{2}(t)\right), i=1,2$, then we have

$$
\frac{\partial}{\partial x_{1}} \varphi_{i}\left(t, x_{1}\right) \geq 0, \quad x_{1}(t)>0, \quad i=1,2
$$

The functional response of predator species $y_{i}(t)$ to immature prey species takes the Holling type III, that is, $p_{i}(i) x_{1}^{2}(t) /\left(k_{i}(t)+x_{1}^{2}(t)\right)$. Holling type III is the third function in which Holling proposed three kinds of functional response of the predator to prey based on numerous experiments for different species. The Holling type form of functional response is intituled prey-dependent model form. It is applied to almost invertebrate that is one of the most extensive applied functional responses.

In [2], Cui and Takeuchi considered the following periodic predator-prey system with a stage structure:

$$
\begin{gathered}
\dot{x}_{1}(t)=a(t) x_{2}(t)-b(t) x_{2}(t)-d(t) x_{1}^{2}(t)-p(t) \phi\left(t, x_{1}\right) x_{1} y(t), \\
\dot{x}_{2}(t)=c(t) x_{1}(t)-f(t) x_{2}^{2}(t), \\
\dot{y}(t)=y(t)(-g(t)+h(t)) \phi\left(t, x_{1}\right) x_{1} y(t)-q(t) y(t),
\end{gathered}
$$

where

$$
0<\phi\left(t, x_{1}\right)<L, \quad \frac{\partial}{\partial x_{1}}\left(\phi\left(t, x_{1}\right) x_{1}\right) \geq 0 \quad\left(x_{1}>0\right)
$$

Different predators usually consume prey in different stage structures. Some predators only prey on immature prey, and some predators only prey on mature prey [5]. Based on system (1.3), we also consider another predator species which also consumes immature prey. Assuming that the predator consumes immature prey according to Holling III functional response while the other predator consumes mature prey also according to the Holling III functional response, we get model (1.1). 
To the best of the authors' knowledge, for the nonautonomous case of predatorprey systems with two predators which consume immature prey and stage structure for prey, whether one could obtain the sufficient and necessary conditions which insure the permanence of the system or not is still an open problem.

The aim of this paper is, by further developing the analysis technique of Cui and Takeuchi [2], to derive a set of sufficient and necessary conditions which ensure the permanence of the system (1.1). The rest of the paper is arranged as follows. In Section 2, we introduce some lemmas and then state the main result of this paper. The result is proved in Section 3.

Throughout this paper, for a continuous $\omega$-periodic function $f(t)$, we set

$$
A_{\omega}(f)=\omega^{-1} \int_{0}^{\omega} f(t) d t
$$

\section{Main Results}

Definition 2.1. The system

$$
\dot{x}=F(t, x), \quad x \in R^{n}
$$

is said to be permanent if there exists a compact set $K$ in the interior of $R_{+}^{n}=\left\{\left(x_{1}, x_{2}, \ldots, x_{n}\right) \in\right.$ $\left.R^{n} \mid x_{i} \geq 0, i=1,2, \ldots, n\right\}$, such that all solutions starting in the interior of $R_{+}^{n}$ ultimately enter $K$ and remain in $K$.

The following lemma can be found in [4].

Lemma 2.2. If $a(t), b(t), c(t), d(t)$, and $f(t)$ are all $\omega$-periodic, then system

$$
\begin{aligned}
& \dot{x}_{1}(t)=a(t) x_{2}(t)-b(t) x_{1}(t)-d(t) x_{1}^{2}(t), \\
& \dot{x}_{2}(t)=c(t) x_{1}(t)-f(t) x_{2}^{2}(t)
\end{aligned}
$$

has a positive $\omega$-periodic solution $\left(x_{1}^{*}(t), x_{2}^{*}(t)\right)$ which is globally asymptotically stable with respect to $R_{+}^{2}=\left\{\left(x_{1}, x_{2}\right): x_{1}>0, x_{2}>0\right\}$.

Lemma 2.3 (see [11]). If $b(t)$ and $a(t)$ are all $\omega$-periodic, and if $A_{\omega}(b)>0$ and $A_{\omega}(a)>0$ for all $t \in R$, then the system

$$
\dot{x}=x[b(t)-a(t) x]
$$

has a positive w-periodic solution which is globally asymptotically stable. 
Theorem 2.4. Suppose that

$$
\begin{gathered}
A_{\omega}\left(-g_{1}(t)+\frac{h_{1}(t)\left(x_{1}^{*}(t)\right)^{2}}{k_{1}(t)+\left(x_{1}^{*}(t)\right)^{2}}\right)>0, \\
A_{\omega}\left(-g_{2}(t)+\frac{h_{2}(t) x_{1}^{*}(t)}{k_{2}(t)+m(t) x_{1}^{*}(t)}+\frac{h_{3}(t)\left(x_{2}^{*}(t)\right)^{2}}{k_{3}(t)+\left(x_{2}^{*}(t)\right)^{2}}\right)>0
\end{gathered}
$$

holds then system (1.1) is permanent, where $\left(x_{1}^{*}(t), x_{2}^{*}(t)\right)$ is the unique positive periodic solution of system (2.2) given by Lemma 2.2.

Theorem 2.5. System (1.1) is permanent if and only if (2.4) holds.

\section{Proof of the Main Results}

We need the following propositions to prove Theorems 2.4 and 2.5. The hypothesis of the lemmas and theorems of the preceding section are assumed to hold in what follows.

Proposition 3.1. There exist positive constants $M_{x}$ and $M_{y}$ such that

$$
\lim \sup _{t \rightarrow+\infty} x_{i}(t) \leq M_{x}, \quad \lim \sup _{t \rightarrow+\infty} y_{i}(t) \leq M_{y}, \quad i=1,2
$$

Proof. Obviously, $R_{+}^{4}$ is a positively invariant set of system (1.1). Given any positive solution $\left(x_{1}(t), x_{2}(t), y_{1}(t), y_{2}(t)\right)$ of $(1.1)$, we have

$$
\begin{gathered}
\dot{x}_{1}(t) \leq a(t) x_{2}(t)-b(t) x_{1}(t)-d(t) x_{1}^{2}(t), \\
\dot{x}_{2}(t) \leq c(t) x_{1}(t)-f(t) x_{2}^{2}(t) .
\end{gathered}
$$

By Lemma 2.2, the following auxiliary equation

$$
\begin{gathered}
\dot{u}_{1}(t)=a(t) u_{2}(t)-b(t) u_{1}(t)-d(t) u_{1}^{2}(t) \\
\dot{u}_{2}(t)=c(t) u_{1}(t)-f(t) u_{2}^{2}(t)
\end{gathered}
$$

has a globally asymptotically stable positive $\omega$-periodic solution $\left(x_{1}^{*}(t), x_{2}^{*}(t)\right)$. Let $\left(u_{1}(t), u_{2}(t)\right)$ be the solution of (3.3) with $u_{i}(0)=x_{i}(0)$. By comparison, we then have

$$
x_{i}(t) \leq u_{i}(t), \quad i=1,2
$$


for $t \geq 0$. By (2.4), we can choose a positive $\varepsilon>0$ small enough such that

$$
\begin{gathered}
A_{\omega}\left(-g_{1}(t)+\frac{h_{1}(t)\left(x_{1}^{*}(t)+\varepsilon\right)^{2}}{k_{1}(t)}\right)>0, \\
A_{\omega}\left(-g_{2}(t)+\frac{h_{2}(t)\left(x_{1}^{*}(t)+\varepsilon\right)}{k_{2}(t)}+\frac{h_{3}(t)\left(x_{2}^{*}(t)+\varepsilon\right)^{2}}{k_{3}(t)}\right)>0 .
\end{gathered}
$$

Thus, from the global attractivity of $\left(x_{1}^{*}(t), x_{2}^{*}(t)\right)$, for the above given $\varepsilon>0$, there exists a $T_{0}>0$ such that

$$
\left|u_{i}(t)-x_{i}^{*}(t)\right|<\varepsilon, \quad t \geq T_{0},
$$

Equation (3.4) combined with (3.6) leads to

$$
x_{i}(t)<x_{i}^{*}(t)+\varepsilon, \quad t>T_{0} .
$$

In addition, for $t \geq T_{0}$, from the third and fourth equations of (1.1) and (3.7) we get

$$
\begin{aligned}
\dot{y}_{1}(t) & \leq y_{1}(t)\left[-g_{1}(t)+\frac{h_{1}(t) x_{1}^{2}(t)}{k_{1}(t)}-q_{1}(t) y_{1}(t)\right] \\
& \leq y_{1}(t)\left[-g_{1}(t)+\frac{h_{1}(t)\left(x_{1}^{*}(t)+\varepsilon\right)^{2}}{k_{1}(t)}-q_{1}(t) y_{1}(t)\right], \\
\dot{y}_{2}(t) & \leq y_{2}(t)\left[-g_{2}(t)+\frac{h_{2}(t) x_{1}(t)}{k_{2}(t)}+\frac{h_{3}(t) x_{2}^{2}(t)}{k_{3}(t)}-q_{2}(t) y_{2}(t)\right] \\
& \leq y_{2}(t)\left[-g_{2}(t)+\frac{h_{2}(t)\left(x_{1}^{*}(t)+\varepsilon\right)}{k_{2}(t)}+\frac{h_{3}(t)\left(x_{2}^{*}(t)+\varepsilon\right)^{2}}{k_{3}(t)}-q_{2}(t) y_{2}(t)\right] .
\end{aligned}
$$

Consider the following auxiliary equation:

$$
\begin{aligned}
& \dot{v}_{1}(t)=v_{1}(t)\left[-g_{1}(t)+\frac{h_{1}(t)\left(x_{1}^{*}(t)+\varepsilon\right)^{2}}{k_{1}(t)}-q_{1}(t) v_{1}(t)\right] \\
& \dot{v}_{2}(t)=v_{2}(t)\left[-g_{2}(t)+\frac{h_{2}(t)\left(x_{1}^{*}(t)+\varepsilon\right)}{k_{2}(t)}+\frac{h_{3}(t)\left(x_{2}^{*}(t)+\varepsilon\right)^{2}}{k_{3}(t)}-q_{2}(t) v_{2}(t)\right] .
\end{aligned}
$$

It follows from (3.5) and Lemma 2.3 that (3.9) has a unique positive $\omega$-periodic solution $y_{i}^{*}(t)>0$ which is globally asymptotically stable. Similarly to the above analysis, there exists a $T_{1}>T_{0}$ such that for the above $\varepsilon$, one has

$$
y_{i}(t)<y_{i}^{*}(t)+\varepsilon, \quad t \geq T_{1}
$$


Let $M_{x}=\max _{0 \leq t \leq \omega}\left\{x_{i}^{*}(t)+\varepsilon: i=1,2\right\}, M_{y}=\max _{0 \leq t \leq \omega}\left\{y_{i}^{*}(t)+\varepsilon: i=1,2\right\}$, then we have

$$
\lim \sup _{t \rightarrow+\infty} x_{i}(t) \leq M_{x}, \quad \lim \sup _{t \rightarrow+\infty} y_{i}(t) \leq M_{y}
$$

This completes the proof of Proposition 3.1.

Proposition 3.2. There exist positive constants $\delta_{i}<M_{x}, i=1,2$, such that

$$
\lim \inf _{t \rightarrow+\infty} x_{i}(t) \geq \delta_{i}, \quad i=1,2
$$

Proof. By Proposition 3.1, there exists $T_{1}>0$ such that

$$
0<x_{i}(t) \leq M_{x} ; \quad 0<y_{i}(t) \leq M_{y} ; \quad t>T_{1}
$$

Hence, from the first and second equations of system (1.1), we have

$$
\begin{gathered}
\dot{x}_{1}(t) \geq a(t) x_{2}(t)-\left(b(t)+\left(\frac{p_{1}(t)}{k_{1}(t)}+\frac{p_{2}(t)}{k_{2}(t)}\right) M_{y}\right) x_{1}(t)-d(t) x_{1}^{2}(t), \\
\dot{x}_{2}(t) \geq c(t) x_{1}(t)-\left(f(t)+\frac{p_{3}(t)}{k_{3}(t)} M_{y}\right) x_{2}^{2}
\end{gathered}
$$

for $t>T_{1}$. By Lemma 2.2, the following auxiliary equation

$$
\begin{aligned}
& \dot{u}_{1}(t)=a(t) u_{2}(t)-\left(b(t)+\left(\frac{p_{1}(t)}{k_{1}(t)}+\frac{p_{2}(t)}{k_{2}(t)}\right) M_{y}\right) u_{1}(t)-d(t) u_{1}^{2}(t) \\
& \dot{u}_{2}(t) \geq c(t) u_{1}(t)-\left(f(t)+\frac{p_{3}(t)}{k_{3}(t)} M_{y}\right) u_{2}^{2}
\end{aligned}
$$

has a globally asymptotically stable positive $\omega$-periodic solution $\left(\tilde{x}_{1}^{*}(t), \tilde{x}_{2}^{*}(t)\right)$. Let $\left(u_{1}(t), u_{2}(t)\right)$ be the solution of (3.15) with $\left(u_{1}\left(T_{1}\right), u_{2}\left(T_{1}\right)\right)=\left(x_{1}\left(T_{1}\right), x_{2}\left(T_{1}\right)\right)$; by comparison, we have

$$
x_{i}(t) \geq u_{i}(t) \quad(i=1,2), t>T_{1} .
$$

Thus, from the global attractivity of $\left(\tilde{x}_{1}^{*}(t), \tilde{x}_{2}^{*}(t)\right)$, there exists a $T_{2}>T_{1}$, such that

$$
\left|u_{i}(t)-\tilde{x}_{i}^{*}(t)\right|<\frac{\tilde{x}_{i}^{*}(t)}{2} \quad(i=1,2), t>T_{2}
$$


Equation (3.17) combined with (3.16) leads to

$$
x_{i}(t)>\delta_{i}=\min _{0 \leq t \leq \omega}\left\{\frac{\tilde{x}_{i}^{*}(t)}{2}\right\}, \quad i=1,2, t>T_{2} .
$$

That is, we have

$$
\lim \inf _{t \rightarrow+\infty} x_{i}(t) \geq \delta_{i}, \quad i=1,2
$$

This completes the proof of Proposition 3.2.

Proposition 3.3. There exists a positive constant $\delta_{y}$ such that

$$
\lim \sup _{t \rightarrow+\infty} y_{i}(t) \geq \delta_{y}, \quad i=1,2
$$

Proof. By assumption (2.4), we can choose a constant $\varepsilon_{0}>0$ and the same constant $\varepsilon$ as in Proposition 3.1 such that

$$
A_{\omega}\left(\psi_{i \varepsilon_{0}}(t)\right)>0, \quad i=1,2
$$

where

$$
\begin{aligned}
\psi_{1 \varepsilon_{0}}(t)= & -g_{1}(t)+\frac{h_{1}(t)\left(x_{1}^{*}(t)-\varepsilon_{0}\right)^{2}}{k_{1}(t)+\left(x_{1}^{*}(t)-\varepsilon_{0}\right)^{2}}-q_{1}(t) \varepsilon_{0} \\
\psi_{2 \varepsilon_{0}}(t)= & -g_{2}(t)+\frac{h_{2}(t)\left(x_{1}^{*}(t)-\varepsilon_{0}\right)}{k_{2}(t)+m(t)\left(x_{1}^{*}(t)+\varepsilon\right)+n(t) \varepsilon_{0}} \\
& +\frac{h_{3}(t)\left(x_{2}^{*}(t)-\varepsilon_{0}\right)^{2}}{k_{3}(t)+\left(x_{2}^{*}(t)-\varepsilon_{0}\right)^{2}}-q_{2}(t) \varepsilon_{0} .
\end{aligned}
$$

Consider the following equation with a parameter $\beta>0$ :

$$
\begin{aligned}
& \dot{x}_{1}(t)=a(t) x_{2}(t)-\left(b(t)+2 \beta\left(\frac{p_{1}(t)}{k_{1}(t)}+\frac{p_{2}(t)}{k_{2}(t)}\right) M_{y}\right) x_{1}(t)-d(t) x_{1}^{2}(t), \\
& \dot{x}_{2}(t)=c(t) x_{1}(t)-\left(f(t)+\frac{p_{3}(t)}{k_{3}(t)} 2 \beta\right) x_{2}^{2} .
\end{aligned}
$$

By Lemma 2.2, (3.23) has a unique positive $\omega$-periodic solution $\left(x_{1 \beta}(t), x_{2 \beta}(t)\right)$, which is globally asymptotically stable. Let $\left(\bar{x}_{1 \beta}(t), \bar{x}_{2 \beta}(t)\right)$ be the solution of (3.23) with initial condition $\bar{x}_{i \beta}(0)=x_{i}^{*}(0), i=1,2$; then, for the above $\varepsilon_{0}$, there exists a $T_{3}>T_{2}$, such that

$$
\left|\bar{x}_{i \beta}(t)-x_{i \beta}(t)\right|<\frac{\varepsilon_{0}}{4}, \quad i=1,2, t>T_{3} .
$$


By continuity of the solution in the parameter, we have $\left(\bar{x}_{1 \beta}(t), \bar{x}_{2 \beta}(t)\right) \rightarrow\left(x_{1}^{*}(t), x_{2}^{*}(t)\right)$ uniformly in $\left[T_{3}, T_{3}+\omega\right]$ as $\beta \rightarrow 0$. Hence, for $\varepsilon_{0}>0$ there exists $\beta_{0}=\beta_{0}\left(\varepsilon_{0}\right)>0$ such that

$$
\left|\bar{x}_{i \beta}(t)-x_{i}^{*}(t)\right|<\frac{\varepsilon_{0}}{4}, \quad t \in\left[T_{3}, T_{3}+\omega\right], 0<\beta<\beta_{0} .
$$

So we have

$$
\left|x_{i \beta}(t)-x_{i}^{*}(t)\right| \leq\left|\bar{x}_{i \beta}(t)-x_{i \beta}(t)\right|+\left|\bar{x}_{i \beta}(t)-x_{i}^{*}(t)\right|<\frac{\varepsilon_{0}}{2}, \quad t \in\left[T_{3}, T_{3}+\omega\right] .
$$

Since $x_{i \beta}(t)$ and $x_{i}^{*}(t)$ are all $\omega$-periodic, we have

$$
\left|x_{i \beta}(t)-x_{i}^{*}(t)\right|<\frac{\varepsilon_{0}}{2}, \quad t \geq 0,0<\beta<\beta_{0}
$$

Choose a constant $\beta_{1}\left(0<\beta_{1}<\beta_{0}, 2 \beta_{1}<\varepsilon_{0}\right)$ and

$$
x_{i \beta_{1}}(t) \geq x_{i}^{*}(t)-\frac{\varepsilon_{0}}{2}, \quad t \geq 0 .
$$

Suppose that the conclusion (3.20) is not true. Then there exists a $Z \in R_{+}^{4}$ such that, for the positive solution $\left(x_{1}(t), x_{2}(t), y_{1}(t), y_{2}(t)\right)$ of (1.1) with an initial condition $\left(x_{1}(0), x_{2}(0), y_{1}(0), y_{2}(0)\right)=Z$, we have

$$
\lim \sup _{t \rightarrow+\infty} y_{i}(t)<\beta_{1}
$$

So there exists a $T_{4}>T_{3}$ such that

$$
y_{i}(t)<2 \beta_{1}, \quad t \geq T_{4}
$$

By applying (3.30), from the first and second equations of system (1.1) it follows that for all $t \geq T_{4}$,

$$
\begin{aligned}
& \dot{x}_{1}(t) \geq a(t) x_{2}(t)-\left(b(t)+2 \beta_{1} \frac{p_{1}(t)}{k_{1}(t)}+2 \beta_{1} \frac{p_{2}(t)}{k_{2}(t)}\right) x_{1}(t)-d(t) x_{1}^{2}(t) \\
& \dot{x}_{2}(t) \geq c(t) x_{1}(t)-\left(f(t)+\frac{p_{3}(t)}{k_{3}(t)} 2 \beta_{1}\right) x_{2}^{2}(t) .
\end{aligned}
$$

Let $\left(u_{1}(t), u_{2}(t)\right)$ be the solution of (3.23) with $\beta=\beta_{1}$ and $u_{i}\left(T_{4}\right)=x_{i}\left(T_{4}\right), i=1$, 2 ; we know that $x_{i}(t) \geq u_{i}(t), t \geq T_{4}, i=1,2$. 
By the global asymptotic stability of $\left(x_{1 \beta_{1}}(t), x_{2 \beta_{1}}(t)\right)$, for the given $\varepsilon=\varepsilon_{0} / 2$, there exists $T_{5} \geq T_{4}$ such that

$$
\left|u_{i}(t)-x_{i \beta_{1}}(t)\right|<\frac{\varepsilon_{0}}{2}, \quad t \geq T_{5}
$$

So we have

$$
x_{i}(t) \geq u_{i}(t)>x_{i \beta_{1}}(t)-\frac{\varepsilon_{0}}{2}, \quad t \geq T_{5}
$$

and hence

$$
x_{i}(t)>x_{i}^{*}(t)-\varepsilon_{0}, \quad t \geq T_{5}
$$

From (3.7) and (3.34), we have

$$
x_{i}^{*}(t)-\varepsilon_{0}<x_{i}(t)<x_{i}^{*}(t)+\varepsilon, \quad t \geq T_{5}
$$

By (3.35) and (1.2), from the third and fourth equations of system (1.1) we have

$$
\dot{y}_{i}(t) \geq \psi_{i \varepsilon_{0}}(t) y_{i}(t), \quad t \geq T_{5}
$$

Integrating (3.36) from $T_{5}$ to $t$ yields

$$
y_{i}(t) \geq y_{i}\left(T_{5}\right) \exp \left\{\int_{T_{5}}^{t} \psi_{i \varepsilon_{0}}(t) d t\right\}, \quad t \geq T_{5}
$$

By (3.21), we know that $y_{i}(t) \rightarrow+\infty$ as $t \rightarrow+\infty, i=1,2$, which is a contradiction. This completes the proof.

Proof of Theorem 2.4. By Propositions 3.2 and 3.3, system (1.1) is uniform weak persistent [2]. From [12, Propositions 3.1 and Theorem 1.3.3], system (1.1) is permanent. This completes the proof of Theorem 2.4 .

Proof of Theorem 2.5. The sufficiency of Theorem 2.5 now follows from Theorem 2.4. We thus only need to prove the necessity of Theorem 2.5. Suppose that

$$
\begin{gathered}
A_{\omega}\left(-g_{1}(t)+\frac{h_{1}(t)\left(x_{1}^{*}(t)\right)^{2}}{k_{1}(t)+\left(x_{1}^{*}(t)\right)^{2}}\right) \leq 0, \\
A_{\omega}\left(-g_{2}(t)+\frac{h_{2}(t) x_{1}^{*}(t)}{k_{2}(t)+m(t) x_{1}^{*}(t)}+\frac{h_{3}(t)\left(x_{2}^{*}(t)\right)^{2}}{k_{3}(t)+\left(x_{2}^{*}(t)\right)^{2}}\right) \leq 0 .
\end{gathered}
$$


We will show that

$$
\lim _{t \rightarrow+\infty} y_{i}(t)=0, \quad i=1,2
$$

In fact, by (3.38), we know that, for any given $0<\varepsilon<1$, there exist $\varepsilon_{1}>0$ and $\varepsilon_{0}>0$ such that

$$
\begin{aligned}
& A_{\omega}\left(-g_{1}(t)+\frac{h_{1}(t)\left(x_{1}^{*}(t)+\varepsilon_{1}\right)^{2}}{k_{1}(t)+\left(x_{1}^{*}(t)+\varepsilon_{1}\right)^{2}}-q_{1}(t) \varepsilon\right) \leq-\frac{\varepsilon}{2} A_{\omega}\left(q_{1}(t)\right) \leq-\varepsilon_{0}, \\
& A_{\omega}\left(-g_{2}(t)+\frac{h_{2}(t)\left(x_{1}^{*}(t)+\varepsilon_{1}\right)}{k_{2}(t)+m(t)\left(x_{1}^{*}(t)+\varepsilon_{1}\right)+n(t) \varepsilon}+\frac{h_{3}(t)\left(x_{2}^{*}(t)+\varepsilon_{1}\right)^{2}}{k_{3}(t)+\left(x_{2}^{*}(t)+\varepsilon_{1}\right)^{2}}-q_{2}(t) \varepsilon\right) \\
& \quad \leq-\frac{\varepsilon}{2} A_{\omega}\left(q_{2}(t)\right) \leq-\varepsilon_{0} .
\end{aligned}
$$

Note that $q_{i}(t)>0$ for $t \geq 0$. Since

$$
\begin{aligned}
& \dot{x}_{1}(t) \leq a(t) x_{2}(t)-b(t) x_{1}(t)-d(t) x_{1}^{2}(t), \\
& \dot{x}_{2}(t) \leq c(t) x_{1}(t)-f(t) x_{1}^{2}(t),
\end{aligned}
$$

we know that, for the given $\varepsilon_{1}$, there exists $T^{(1)}>0$ such that

$$
x_{i}(t) \leq x_{i}^{*}(t)+\varepsilon_{1}, \quad t \geq T^{(1)}, i=1,2 .
$$

By (3.40), (1.2), and (3.42), we have

$$
\begin{aligned}
& A_{\omega}\left(-g_{1}(t)+\frac{h_{1}(t) x_{1}^{2}(t)}{k_{1}(t)+x_{1}^{2}(t)}-q_{1}(t) \varepsilon\right) \leq-\varepsilon_{0}, \quad t \geq T^{(1)} \\
& A_{\omega}\left(-g_{2}(t)+\frac{h_{2}(t) x_{1}(t)}{k_{2}(t)+m(t) x_{1}(t)+n(t) \varepsilon}+\frac{h_{3}(t) x_{2}^{2}(t)}{k_{3}(t)+x_{2}^{2}(t)}-q_{2}(t) \varepsilon\right) \leq-\varepsilon_{0}, \quad t \geq T^{(1)} .
\end{aligned}
$$

We now show that there must exist $T^{(2)}\left(>T^{(1)}\right)$ such that $y_{i}\left(T^{(2)}\right)<\varepsilon$. Otherwise, by (3.43), we have

$$
\begin{aligned}
& \varepsilon \leq y_{1}(t) \leq y_{1}\left(T^{(1)}\right) \exp \left\{\int_{T^{(1)}}^{t}\left[-g_{1}(s)+\frac{h_{1}(s)\left(x_{1}(s)\right)^{2}}{k_{1}(s)+\left(x_{1}(s)\right)^{2}}-q_{1}(s) \varepsilon\right] d s\right\} \rightarrow 0, \quad t \rightarrow+\infty \\
& \varepsilon \leq y_{2}(t) \leq y_{2}\left(T^{(1)}\right) \exp \left\{\int_{T^{(1)}}^{t}\left[-g_{2}(s)+\frac{h_{2}(s) x_{1}(s)}{k_{2}(s)+m(s) x_{1}(s)+n(s) \varepsilon}+\frac{h_{3}(s) x_{2}^{2}(s)}{k_{3}(s)+x_{2}^{2}(s)}-q_{2}(s) \varepsilon\right] d s\right\} \\
& \longrightarrow 0, \quad t \longrightarrow+\infty .
\end{aligned}
$$


This implies $\varepsilon \leq 0$, which is a contradiction.

$$
\begin{aligned}
& M_{1}(\varepsilon)=\max _{0 \leq s \leq \omega}\left\{-g_{1}(s)+\frac{h_{1}(s)\left(x_{1}(s)\right)^{2}}{k_{1}(s)+\left(x_{1}(s)\right)^{2}}-q_{1}(s) \varepsilon\right\}, \\
& M_{2}(\varepsilon)=\max _{0 \leq s \leq \omega}\left\{-g_{2}(s)+\frac{h_{2}(s) x_{1}(s)}{k_{2}(s)+m(s) x_{1}(s)+n(s) \varepsilon}+\frac{h_{3}(s) x_{2}^{2}(s)}{k_{3}(s)+x_{2}^{2}(s)}-q_{2}(s) \varepsilon\right\} .
\end{aligned}
$$

We know that $M_{i}(\varepsilon)$ is bounded (Proposition 3.1 given). We now show that

$$
y_{i}(t) \leq \varepsilon \exp \left\{M_{i}(\varepsilon) \omega\right\}, \quad t \geq T^{(2)}, i=1,2
$$

Otherwise, there exists $T^{(3)}>T^{(2)}$ such that

$$
y_{i}\left(T^{(3)}\right)>\varepsilon \exp \left\{M_{i}(\varepsilon) \omega\right\}
$$

By the continuity of $y_{i}(t)$, there must exist $T^{(4)} \in\left(T^{(2)}, T^{(3)}\right)$ such that $y_{i}\left(T^{(4)}\right)=\varepsilon$ and $y_{i}(t)>\varepsilon$ for $t \in\left(T^{(4)}, T^{(3)}\right]$. Let $P_{1}$ be the nonnegative integer such that $T^{(3)} \in\left(T^{(4)}+P_{1} \omega, T^{(4)}+\left(P_{1}+1\right) \omega\right]$; by (3.43) we have

$$
\begin{aligned}
\varepsilon \exp \left\{M_{1}(\varepsilon) \omega\right\} & <y_{1}\left(T^{(3)}\right) \\
& <y_{1}\left(T^{(4)}\right) \exp \left\{\int_{T^{(4)}}^{T^{(3)}}\left[-g_{1}(t)+\frac{h_{1}(t)\left(x_{1}(t)\right)^{2}}{k_{1}(t)+\left(x_{1}(t)\right)^{2}}-q_{1}(t) \varepsilon\right] d t\right\} \\
& =\varepsilon \exp \left\{\int_{T^{(4)}}^{T^{(4)}+P_{1} \omega}+\int_{T^{(4)}+P_{1} \omega}^{T^{(3)}}\right\}\left[-g_{1}(t)+\frac{h_{1}(t)\left(x_{1}(t)\right)^{2}}{k_{1}(t)+\left(x_{1}(t)\right)^{2}}-q_{1}(t) \varepsilon\right] d t \\
& \leq \varepsilon \exp \left\{\int_{T^{(4)}+P_{1} \omega}^{T^{(3)}}\left[-g_{1}(t)+\frac{h_{1}(t)\left(x_{1}(t)\right)^{2}}{k_{1}(t)+\left(x_{1}(t)\right)^{2}}-q_{1}(t) \varepsilon\right] d t\right\} \\
& <\varepsilon \exp \left\{M_{1}(\varepsilon) \omega\right\},
\end{aligned}
$$




$$
\begin{aligned}
\varepsilon \exp \left\{M_{2}(\varepsilon) \omega\right\}<y_{2}\left(T^{(3)}\right) & \\
< & \quad y_{2}\left(T^{(4)}\right) \exp \left\{\int _ { T ^ { ( 4 ) } } ^ { T ^ { ( 3 ) } } \left[-g_{2}(t)+\frac{h_{2}(t) x_{1}(t)}{k_{2}(t)+m(t) x_{1}(t)+n(t) \varepsilon}\right.\right. \\
& =\varepsilon \exp \left\{\int_{T^{(4)}}^{T^{(4)}+P_{1} \omega}+\int_{T^{(4)}+P_{1} \omega}^{T^{(3)}}\right\}\left[-g_{2}^{2}(t)+\frac{h_{2}(t) x_{1}(t)}{k_{2}(t)+m(t) x_{1}(t)+n(t) \varepsilon}\right. \\
& \left.+\frac{h_{3}(t) x_{2}^{2}(t)}{k_{3}(t)+x_{2}^{2}(t)}-q_{2}(t) \varepsilon\right] d t \\
\leq & \varepsilon \exp \left\{\int_{T^{(4)}+P_{1} \omega}^{T^{(3)}}\left[-g_{2}(t)+\frac{h_{2}(t) x_{1}(t)}{k_{2}(t)+m(t) x_{1}(t)+n(t) \varepsilon}+\frac{h_{3}(t) x_{2}^{2}(t)}{k_{3}(t)+x_{2}^{2}(t)}-q_{2}(t) \varepsilon\right] d t\right\} \\
& <\varepsilon \exp \left\{M_{2}(\varepsilon) \omega\right\},
\end{aligned}
$$

which is a contradiction. This implies that (3.46) holds. We then conclude, by the arbitrariness of $\varepsilon$, that $y_{i}(t) \rightarrow 0$ as $t \rightarrow+\infty, i=1,2$. This completes the proof of Theorem 2.5.

\section{References}

[1] J. Cui and X. Song, "Permanence of predator-prey system with stage structure," Discrete and Continuous Dynamical Systems —Series B, vol. 4, no. 3, pp. 547-554, 2004.

[2] J. Cui and Y. Takeuchi, "A predator-prey system with a stage structure for the prey," Mathematical and Computer Modelling, vol. 44, no. 11-12, pp. 1126-1132, 2006.

[3] X.-A. Zhang, L. Chen, and A. U. Neumann, "The stage-structured predator-prey model and optimal harvesting policy," Mathematical Biosciences, vol. 168, no. 2, pp. 201-210, 2000.

[4] J. Cui, L. Chen, and W. Wang, "The effect of dispersal on population growth with stage-structure," Computers \& Mathematics with Applications, vol. 39, no. 1-2, pp. 91-102, 2000.

[5] C.-Y. Huang, M. Zhao, and L.-C. Zhao, "Permanence of periodic predator-prey system with two predators and stage structure for prey," Nonlinear Analysis: Real World Applications, vol. 11, no. 1, pp. 503-514, 2010.

[6] H. Baek, "Species extinction and permanence of an impulsively controlled two-prey one-predator system with seasonal effects," BioSystems, vol. 98, no. 1, pp. 7-18, 2009.

[7] F. Chen, "Permanence of periodic Holling type predator-prey system with stage structure for prey," Applied Mathematics and Computation, vol. 182, no. 2, pp. 1849-1860, 2006.

[8] W. Wang and L. Chen, "A predator-prey system with stage-structure for predator," Computers $\mathcal{E}$ Mathematics with Applications, vol. 33, no. 8, pp. 83-91, 1997.

[9] W. Yang, X. Li, and Z. Bai, "Permanence of periodic Holling type-IV predator-prey system with stage structure for prey," Mathematical and Computer Modelling, vol. 48, no. 5-6, pp. 677-684, 2008.

[10] X. Zhang, L. Chen, and A. U. Neumann, "The stage-structured predator-prey model and optimal harvesting policy," Mathematical Biosciences, vol. 168, no. 2, pp. 201-210, 2000.

[11] X.-Q. Zhao, "The qualitative analysis of $N$-species Lotka-Volterra periodic competition systems," Mathematical and Computer Modelling, vol. 15, no. 11, pp. 3-8, 1991.

[12] X.-Q. Zhao, Dynamical Systems in Population Biology, vol. 16 of CMS Books in Mathematics, Springer, New York, NY, USA, 2003. 


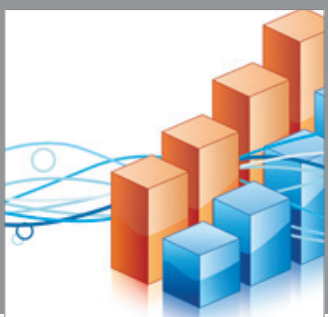

Advances in

Operations Research

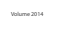

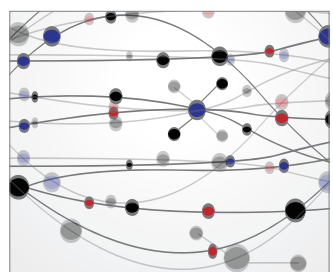

\section{The Scientific} World Journal
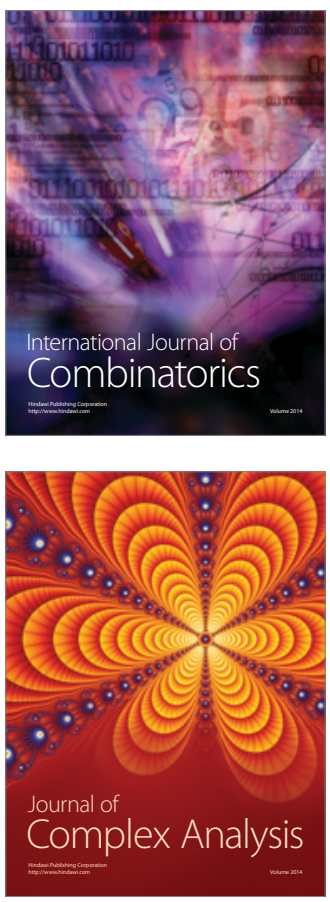

International Journal of

Mathematics and

Mathematical

Sciences
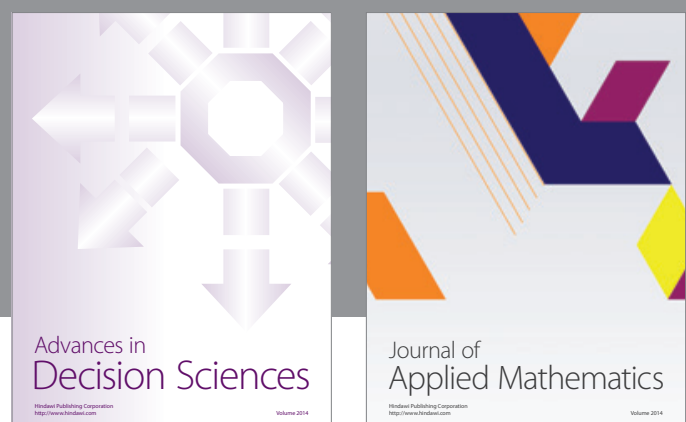

Journal of

Applied Mathematics
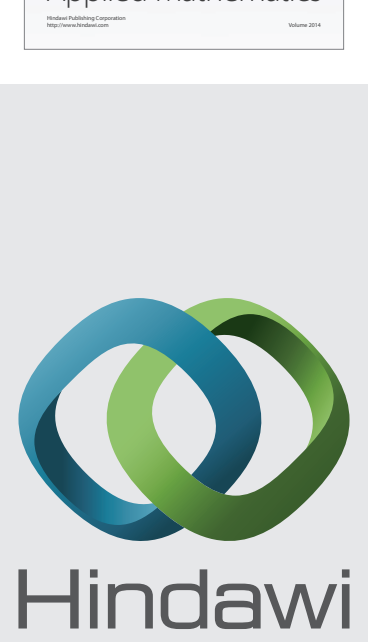

Submit your manuscripts at http://www.hindawi.com
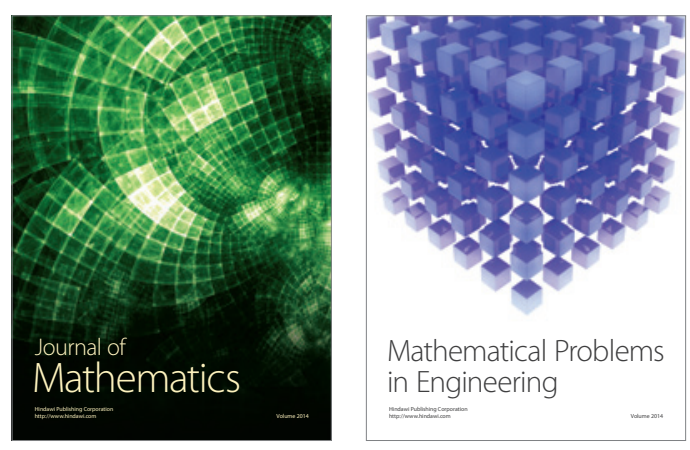

Mathematical Problems in Engineering
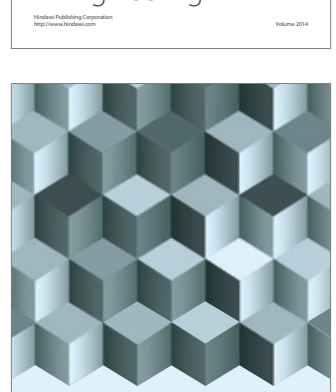

Journal of

Function Spaces
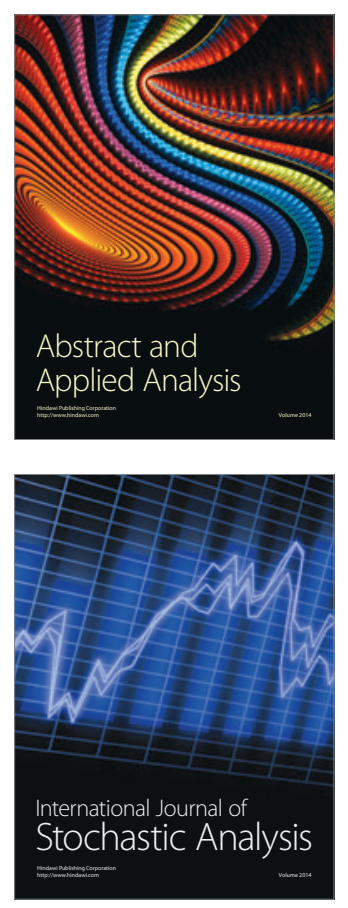

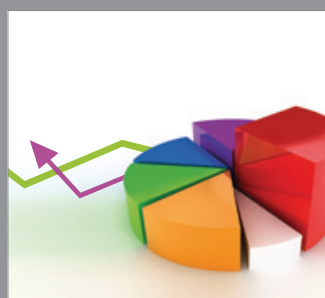

ournal of

Probability and Statistics

Promensencen
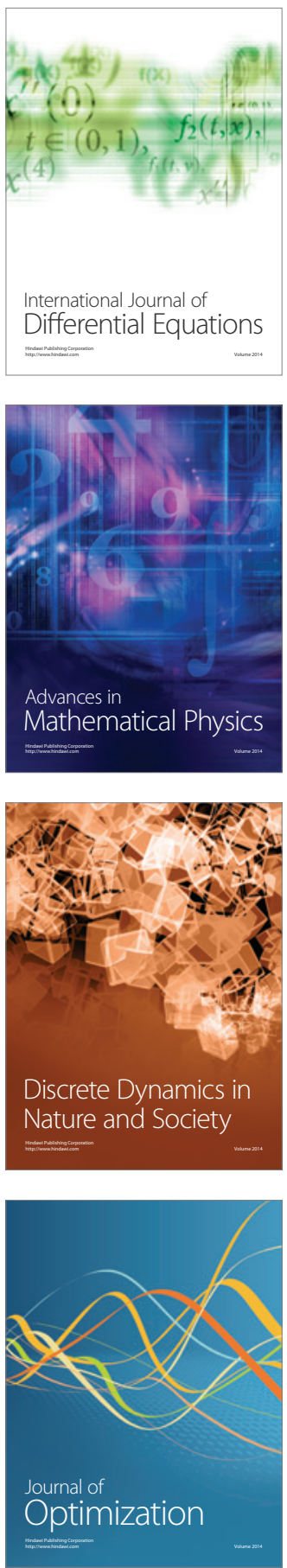